\title{
M. Regula
}

\section{REMARQUES SUR n'avoir pas un sou vaillant (Contribution à la méthode de l'analyse syntactique)}

Dans un article paru dans la Zeitschrift für französische Sprache und Literatur, LVIII, S. 22-27, M. St. Lyer a tâché d'expliquer la forme et la fonction de vaillant. Comme, à notre avis, son étude a besoin d'être complétée par des exemples tout particuliers, nous nous sommes proposé pour tâche de reprendre ce sujet.

Avant d'examiner les exemples rassemblés par l'auteur et quelques autres, nous voulons passer en revue les moyens d'expression pour l'indication de la valeur, du prix et de la quantité en modifiant tant soit peu l'aperçu de l'auteur. L'uobliquus pretii", sorti de la confusion de l'accusatif et de l'ablatif pretii, peut être exprimé:

1. par un substantif de quantité avec ou sans préposition, 2. par le neutre de l'adjectif, ou du pronom, ou par l'adverbequantitatif, 3. par une proposition relative périphrasant le concept de mesure. La conclusion que l'adverbe de quantité ne pourrait jamais avoir la terminaison -ment demande une rectification. La vie du language nous met en garde contre de telles généralisations précipitées, puisqu'elle ne les dément que trop souvent. En dépit de la thèse établie par l'auteur il y a des adverbes de mesure en -ment, $\mathrm{p}$. ex.: La victoire nous a coûté ch èr e ment (à côté de cher); - Nous venarons notre vie ch èr e ment. On rencontre énormément, abondamment, infiniment, diablement, etc., concurrents affectifs de beaucoup, qui renferment sans doute une nuance de quantité: avoir enormément de travail; il y a suffis amment à voir; vous risquez $d i a b l$ e ment (Molière), phrases, où le syntagme adverbe + nom constitue plus ou moins un «objet de mesure» (v. Regula, Grundlegung und Grundprobleme der Syntax, §29, II, 4, p. 93).

Quant à la fonction syntactique de $v$ a $i l l$ a $n t$, elle dépend de l'es $\mathrm{p}$ è ce de connexion. Il serait tout à fait erroné de voir a priori dans celui-la un winstrumentalis pretii» ou un cas zéro, présenté par les expressions explicatives couleur, genre, style dans des combinaisons telles que celles-ci: une étoffe couleur feuille morte, un château styl.e Renaissance, etc. C'est que vaillant peut avoir plusieurs valeurs syntactiques:

1. N'a ta moillier n'a dame qu'as veüd // n'en vanteras el regne dont tu fus, vaillant denier que m'i aies tolut, // ne fait damage ne de mei ne d'altrui (Rol., $1960 \mathrm{ss}$.). K. Voreztsch explique le syntagme en question par: "akkusativ des gerunđiums 'das, was einen Heller wert ist - eines hellers wert'《 (Altfranzösisches Lesebuch, 28, p. 79). 
Il ne vous aime vaillant un oef pelé (Mariage Guillaume): ici, vaillant indique la valeur générale du syntagme un oef pelé. M. Lyer voit dans vaillant un prédicatif, interprétation qui nous semble trop artificielle. Courton grand risque à interpréter vaillant comme expression condensée de d'un amour vaillant ( 1 ablativus modi interne ${ }^{2}$ ) ou tout simplement comme adverbiel ( «avec la valeuru)? Un cas pareil nous est offert dans le passage du Roman de la Rose que voici: Sans moi prisier un of f vaillant, où, à notre surprise, $M$. Lyer suppose à juste titre un «instrumentalis pretiik, c'e.-à $d$. un adverbiel de mesure ( Ne prise sa menache vailissant un caudel. (Gui de Nanteuil). Car il ne crient autrui menace N'autrui coroz vaillant deus foles (Roman de: Renart). Fole n'a pas la signification de «fable», mais provient de "fabulı, diminutif de faba.

Dans son traité Die Verwendung des Gerundiums und des Partiaips praesentis im Französischen, Göttinger Dissertation, 1912, E. Mönch parle d'un "accusativus pretii», tandis qu'il attribue au gérondif la valeur d'un «instrumentalis pretii», mais qui ne s'applique pas à tous les emplois de la forme nominale.

2: Avec les verbes cités par l'auteur (porter, retenir, toldre, laissier, gaaignier, chargier, perdre, donner, prendre, auxquels ils faut ajouter avoir) l'indication de prix ne peut avoir d'autre fonction que celle d'objet direct, le sens général du verbe valoir exigeant un objet interne de spécialisation. Premièrement Colin Laurens, Girart Gossouyn et Jehan Marcean, Dépourvus de biens, de parens, Qui n'ont vaillant l'ance d'ung seau (Villon, Lais XXVI, 4). Et ne deust il quoir vaillant ung pigne (id., Grand Testament, 1248). Repos éternel donne a cil, Sire, et clarté perpétuelle, Qui vaillan $t$ plat ni escuelle $N$ 'eut oncques $n$ 'un brain de percil (ib., 1894 sq.).

3. Vaisselle e dras e or e argent $y$ ont doné vaillant mil mars (Ducs de Normandie, 9974) avec un complément déterminatif détaché ("Fernattribut») au sens de: "qui valent», "en valeur de...»,- Pour un escot huit sols montant (Villon, Petit Testament, 86), où il s'agit d'un com:

${ }^{1} \mathrm{Cp} .:$ Mais tant l'ama Erec d'a mors, que d'armes mais ne li chaloit (Erec, 2434, cité par E. Gamillscheg, Hist. franz. Syntax, p. 517).

${ }^{2} \mathrm{Cp}$. en lat.: honesta morte mori (Sénèque).

${ }^{3}$ Le fameux vers de Malherbe: Et rose, elle a vécu ce que vivent les roses renferme aussi un objet interne de mesure ou d'espace, qui, dans la contexture spirituelle de la phrase, produit son effet.

${ }^{4} \mathrm{Cp}$.: Il s'en soucie a utant que d'une noix. Avec un geste explicatif: Je me souciais de vingt canons comme de cela (E. About, Le Roi des montagnes). Lat. $h u i u s$ ("gen. pretii») non faciam (locution deictique chez Térence, $A d$., 278). Il n'a pas $c$ a d'amour propre.

$\mathrm{Cp}$. l'origine de la négation bipartite (ne...mie, goutte, brin, mot, ombre, soupçon, etc., dont les germes sont à chercher déjà en latin, p. ex.: Sisiphus versat saxum sudans nitendo neque proficit hilum (Cicéron, disp. Tusc., I, 10). Quinque dies aquam in os suum non coniecit, non micam panis (Pétrone, 42). Non hodie buccam panis invenire potui. (ib.). L'ancien français possédait pour objets de mesure festu, denier, bouton, ail, guant. 
plément déterminatif normal. Même pour cet emploi de vaillant il n'y a pas de quoi lui contester la nature de gérondif ou de participe, que A. Stimming, E. Mönch, Th. Kalepky, J. Haas, K. Sneyders de Vogel, resp. A. Tobler, E. Lerch, O. Bloch et W. v. Wartburg lui ont attribuée.'

4. Del grand duel que li autre font, Ne li est le vallant de us nois (Perceval, 39373), avec la forme analytique du prédicat ( = verbe abstrait + composant sémantique). Li vaillisant d'un sol denier Ne l'esteit mie lessié (Roman de Troie, 28436): l'indication de valeur est marquée comme complément déterminatif. N'ayants rien en le $u r$ vaillant par où se produire, il cherchent à se présenter par une valeur estrangiere [les plagiaires] (Montaigne, I, 457). Cesar s'endebte d'un milion d'or oultre son $v$ a $i l l$ a $n t$ (id., I, 343). Les déterminatifs (article, adjectif possessif ou pronom personnel adjectif) donnent à la forme vaillant un caractè $\mathrm{re} \mathrm{s} \mathrm{u} b$ stantival plus prononcé (= "la valeur»).

Il nous reste maintenant à expliquer forme et valeur syntactique de vaillant dans notre locution. En refusant l'explication de Tobler, qui considère vaillant comme participe substantivé, M. Lyer s'appuie sur l'objection faite par E. Mönch qu'un tel au sens d'un nom abstrait ne se rencontre qu'à partir du dix-septième siècle, et décrète: "Vaillant est donc un adjectif provenant directement du participe latin valens et n'ayant rien de commun avec le participe présent du verbe valoir: à cause de sa nature purement adjective il peut être substantifié avec le sens abstrait "la valeur»». Conclusion hasardée, à ce qu'il nous semble. Que des participes ou des gérondifs substantivés ne se trouvent pas avant le dix-septième siècle, c'est une assertion facile à réfuter. Un examen de la Chanson de Roland fait à la hâte nous offre cuntenart (v. 118, 3116) = «contenance»; semblant (v. 270) = «facon« (ou "avis»?), guarant (v. 290) = "garantie»; vostre veiant (v. 287) =' «en votre présence»; en tut vostre vivant (v. 872). En outre on trouve: Al terme de son moriant. n'ot le reis eu nul enfant (Brut, 5390). N'ot pas fui une lieue t e n a $n t$ (= "la teneur», "distance») (Aliscans, 85). Remenant = "reste» (Villon, Grand Testament, v. 749); tout d'ung tenant = «en bloc» (ib. 751). Tobler cite dans VB, $\mathrm{I}$, 52: le bi e $n$ vi e g $n$ an $t$ ( = "la bienvenue») fust grant entre eulx. ${ }^{2}$

Ici se pose de nouveau la question: Participe ou gérondif? Nous penchons pour l'opinion de Th. Kalepky, qui s'est décidé pour le gérondif même quand celui-ci figure comme complément déterminatif. Mais comme il ne s'agit que d'hypothèses, l'éthique scientifique interdit d'en ériger l'une en dogme; c'est pourquoi on admettra aussi la diagnose des savants Tobler et Lerch.

1 L'équivalence du gérondif et du participe présent se rencontre déjà chez Salluste et Tacite: Bocchus seu reputando (= reputans) seu admonitus... quinque delegit (bell. Iug. 103, 2); impetu pervagatum incendium plana primum, deinde in edita as $s$ rgens et rursus inferiora pop ula $n$ do anteiit remedia velocitate mali ( $A$ nn., $\mathrm{XV}, 38$ ); socors ingenium eius in contrarium trahens callidumque et simulatorem in te $r p$ re t a $n d$ o (ib., XIII, 47).

${ }^{2}$ Gamillscheg cite encore: avenant $=$ "convenance», convenant $=$ uconvention», pesant $=$ «poids». 

rondif ${ }^{\text {: }}$

Qu'il nous soit permis de donner un aperçu des emplois figés du gé-

I. en forme conjointe:

1. comme adverbiel (indication de manière):

payer (verser) argent comptant, locution dont l'analyse offre deux. possibilités: a) comptant au sens actif; b) = «qui se compte» (voix. moyenne).

2. comme complément sémantique du verbe principal dans la composition syntactique:

faire semblant (= "feindre»).

II. en fonction de "participium coniunctum":

chemin faisant (it. cammin facendo), ce faisant, quoi faisant, cer disani.

III. à l'intérieur d'un circonstanciel (annexe du "phénomène»"):

1. a) à midi tapant, à deux heures sonnant; main t e n a $n t$ (geste decommandement). Comparant les tournures du vieux français a soleil levant, al coc chantant, à la lune luisant, après l'aubé aparant. ${ }^{3}$

b) à son corps défendant ( = «en légitime défense»). L'ancien francais connaissait un emploi vivant du gérondif prépositionnel: eust grant peor de la teste perdant (Jérusalem.). A cel eve passant (Rou, 2, 3806) etc.

IV. en fonction d'un "ablativus absolutus» sans sujet grammatical ou '̀. sujet indéterminé:

littéralement (généralement) parlant (it. generalmente parlando): "judicatif" (prise de position envers le contenu de la phrase). On. pourrait penser aussi à une imitation savante du "dativus iudicantis»: in universum aestimanti (Tacite, Germ., 6) - S'agissant; donnant donnant; vivant mourant; dormant veillant.

V. en tant que substantif:

1. dans des prépositionnels:

$\grave{a}$ bon escient (= "sciemment»), à monescient (= "que je sache», "autant que je sais»); de mon vivant; se mettre (se dresser) sur son séant.

${ }^{\prime} \mathrm{Cp}$. St. Skerlj, Syntaxe du participe présent et du gérondif en vieil italien, $\S \S 848-852$.

2. C'est ainsi que A. Jaeggi appelle le noyau de la-phrase ( La préposition dans les rapports abstraits; Bern, 1956; p. 16).

${ }^{3}$ Puisque les prédicats condensés (ou périphériques) sont formés de verbes neutres, les substantifs ont la fonction de sujets logiques au sein du circonstanciel. Expression co n crèt e pour: "au lever du soleil», "au chant du coq», "au clair de lune», "après l'apparition de l'aube». 
2. valoir son pesant d'or, permutation de: avoir son or pesant. ${ }^{1}$ Retournons, après cette digression, à la locution n'avoir pas un sou vailIant, qui a pour variantes $n^{\prime}$ 'voir pas un écu vaillant, n'avoir pas vaillant un. quart d'écu. Les deux premières tournures s'avèrent plus anciennes, le sousobjet dépendant de vaillant étant antéposé, à moins qu'on n'interprète vaillant comme détermination explicative ( «en valeur», "en capital») ou même comme prédicatif. Un passage très intéressant à ce point de vue se trouve dans Souvestre, L'Oncle d'Amérique. Le voici: Je l'ai rachetée [la montre] avec tout ce que javais vailla n,t. Une diagnose exacte du rôle syntactique de vaillant fait pas mal de difficultés. Ce qui est sûr, c'est que vaillant est en connexion de sens avec tout. Pour la fonction grammaticale, il y a deux explication possibles:

1. Vaillant peut être interprété comme prédicatif; cp. J'ai mon gars $s$ olda t comme toi (P. Déroulède, Le bon Gîte). Il est vrai que le prédicatif avec avoir est en général introduit par la préposition pour.

2. Vaillant serait la juxtaposition d'un complément déterminatif détaché ("Fernattribut») au lieu de la forme régulière avec de, p. ex.: [Condé] a promis et engagé... tout ce qu'il a de vaillant ${ }^{3}$ de faire la paix à Bordeaux (Gui Patin, Nouv. lett., t. I, p. 234).

La tournure n'avoir plus rien vaillant n'admet pas moins de trois analyses:

1. rien vaillant: vaillant (o b j t di $\mathrm{rect}$ ) + rien (o bjet a e mesu $\mathrm{r}$ e en dépendance de vaillant) $=$ "qui ne vaille rien». Cp. un rien vaillant, synonyme vulgaire de "vaurien», "fainéant». Pour le genre neutre, cp. un pas grand'chose.

2. rien vaillant: rien qui vaille. (cp.: Je n'ai rien fait. qui vaille). Vaillant serait en ce cas détermination qualificative (en forme 'd'adjectif).

3. rien vaillant: pour rien de vaillant. Pour la juxtaposition il est à comparer avec rien tel, rien autre chose, personne autre; force bouteilles, au temps jadis, à huit heures jaquemart.

\section{Conclusion.}

1. Le gérondif (ou participe) vaillant, exposant d'une indication de valeur, s'emploie comme "d é te r $\mathrm{m}$ in a $\mathrm{n}$ t» en fonction de complément déter-

${ }^{1}$ Pour cette inversion constructive il y a assez d'exemples: boire à $m$ ê $m e$ d'une bouteille < ̀̀ même la bouteille; à moins de + nom ou infinitif $<$ moins + nom, remontant à un ablativus absolutus, p. ex.: me minus uno; à sec de toile, à étanche d'eau, à ras à sol, à plat de, sabre, à changé de visée (Molière, Tartuffe, II, 4), du meilleur de mon coeur (de mon àme).

${ }^{2}$ Vaillant antéposé permet la conclusion que l'indication de valeur a la fonction d'objet.

3. Cet exemple revèle la contradiction entre le terme grammatical et la réalité. Le soi-disant "génitif partitif» est dans ce cas "génitif du tout) au sens propre. Ce terme est d'ailleurs le seul juste pour tous les cas où le nucléus exprime vraiment une partie du tout, p. ex.: Des trois les deux sont morts. Par contre, des cas comme beaucoup (peu, assez, une foule) de gens contiennent un génitif de matière. 
minatif, ou comme "d éterminé» en fonction de terme régissant. ${ }^{\mathrm{t}}$ Suivant le cas, il a le caractère de nom ou d'adjectif.

2. En connexion avec vaillant, le complément de valeur remplit la fonction d'objet direct et de complément déterminatif.

3. Le syntagme: vaillant + indication de valeur peut figurer comme objet direct, comme complément déterminatif commun ou détaché, et comme sujet.

Quoique notre étude n'ait pas abouti à une solution définitive du pro. blème, nous croyons pourtant avoir démontré les différents aspects sous lesquels il faut examiner un phénomène compliqué de syntaxe.

Graz, janvier 1964.

\section{POVZETEK}

Povod za nasleảnje Opazke o frazi "n'avoir pas un sou vaillant» je dal članek Stanislava Lyer-a - objavljen $\vee$ Zeitschrift für französische Sprache und Literatur, LVIII, str. $22-27-0$ isti konstrukciji. Prof. Regula se ne strinja z Lyerovo razlago, ki trdi, da je wvaillant (torej) adjektiv, ki izhaja neposredno iz lat. participa valens in nima nič skupnega s participom sed. časa glagola valoir: zaradi svoje čisto adjektivne narave se lahko substantivira $\mathrm{z}$ abstraktnim pomenom 'la valeur'u. Prof. Regula ne namerava malega, toda zelo zaplétenega problema, ki ga zadaja konstrukcija "n'avoir pas un sou vaillantk, že tukaj dokončno rešiti, pač pa hoče nedovoljni inventar, na katerega se opira Lyer, razširiti z novimi, značilnimi primeri, ki kažejo vaillant $v$ raznih, med seboj očitno sorodnih, a nikakor ne identičnih situacijah, in $s$ tèm dati trdnejšo podlago za pravilno ugotovitev njegove resnične vrednosti in sintaktične funkcije, hkrati pa $\mathrm{z}$ analizo primerov olajšati končno sodbo.

Funkcija se praviloma zrcali $v$ specifični povezavi oblike vaillant $\mathrm{z}$ ostalimi deli stavka in bi se torej morala dati določiti. Težava pa je $v$ tem, da je povezav več in da dopuščajo nekatere po dve ali tri interpretacije. Naj navedemo tu nekaj glavnih tipov (francoski tekst daje zanje še mnogo drugih primerov): "Il ne vous aime vaillant un oef pelé: (Mariage Guillaume); - "Premièrement Colin Laurens, Girart Gossouyn et Jehan Marceau, Dépourvus de biens, de parens, Qui n'ont vaillant l'ance d'ung seauk (Villon); - "Vaisselle e dras e or e argent y ont doné vaillant mil mars (Ducs de Normandie); - „Cesar s'endebte d'un milion a'or oultre son vaillant" (Montaigne); - "Je l'ai rachetée /la montre/ avec tout ce que j'avais vaillant« (Souvestre, L'Oncle d'Amérique). Pisec analizira in grupira te različne rabe in pride do naslednjih zaključkov: Ne prisvaja si pravice, da bi razsodil staro vprašanje, ali je ta vaillant, ki služi kot eksponent $\mathrm{v}$ označbi vrednosti, gerundij ali particip; pač pa vidi, da ima včasih značaj substantiva, včasih adjektiva, in predvsem, da se lahko rabi bodisi kot ndeterminantk v determinativni funkciji, ali pa. kot sdeterminirano" $\mathrm{v}$ funkciji nadrejene besede (od katere je izraz za vrednost gramatično odvisen); - dalje, da ima izraz za vrednost, ki je odvisen od besede vaillant, nasproti le-temu' funkcijo direktnega objekta in determinantnega dopolnila; - in še, da celotna sintagma wvaillant + izraz za vrednostu lahko predstavlja ali direktni objekt, ali navadno ali pa »oddaljenow determinantno dopolnilo (atribut //Fernattribut《/), ali pa subjekt.

Morda ta analiza in opredelitev funkcij besede vaillant lahko služi kot primer metode, s kakršno velja preiskovati takle zapleten sintaktični pojav.

${ }^{1}$ C'est un paradoxe de syntaxe que le terme subordonné détermine le terme régissant. 